\title{
Temperature influence on optical charging of self-assembled InAs/GaAs semiconductor quantum dots
}

\author{
K. F. Karlsson, ${ }^{\text {a) }}$ E. S. Moskalenko, ${ }^{\text {b) }}$ P. O. Holtz, and B. Monemar \\ Department of Physics and Measurement Technology, Linköping University, S-581 83 Linköping, Sweden \\ W. V. Schoenfeld, J. M. Garcia, and P. M. Petroff \\ Materials Department, University of California-Santa Barbara, Santa Barbara, California 93106
}

(Received 25 January 2001; accepted for publication 9 March 2001)

\begin{abstract}
It is demonstrated that the photoluminescence spectra of single self-assembled InAs/GaAs quantum dots are very sensitive to excitation energy and crystal temperature. This is qualitatively explained in terms of the effective diffusivity of photogenerated particles, which affects the capture probability of the quantum dot. As a consequence, this opens the possibility of controlling the average number of excess electrons in the quantum dot by optical means. This technique may be used as a simple tool to create and study charged exciton complexes without any specially fabricated samples. (C) 2001 American Institute of Physics. [DOI: 10.1063/1.1370547]
\end{abstract}

In the last few years, significant research effort has been focused on self-assembled semiconductor quantum dots (SAQDs), which has led to growth ${ }^{1,2}$ and characterization ${ }^{3}$ techniques that make it possible to study single SAQDs. Photoluminescence (PL) spectroscopy with high spatial resolution performed on single quantum dots (QDs) makes it possible to reveal the fine structure in the individual dot spectrum. This is in contrast to studies of a QD ensemble, in which the fine structure normally is concealed by the inevitably inhomogeneous broadening. Simultaneously, the development of theoretical models, ${ }^{4}$ considering many particle interactions, now enables a more advanced study of multiparticle complexes in QDs. Since the particles in a QD are confined into a spatially limited region, there is a strong interaction between them. The addition of one or more particle(s), therefore, changes the emission energies in the QD spectrum dramatically. Consequently, it is important to understand multiparticle effects because they determine the performance of electronic devices such as QD lasers and single-electron transistors.

The QD becomes charged when it is filled with a nonequal amount of electrons and holes. Essentially, two approaches have recently been used to study charged exciton complexes in single QDs. One method is to use specially designed samples ${ }^{5}$ containing an $n$-doped layer with contacts. The number of electrons in the QD can then be tuned by an applied external voltage. This gives the possibility to control the average number of excess electrons in the dot. However, the applied voltage causes a shift of the entire spectrum and deforms the wave functions, which eventually leads to a relative shift of the interaction energies. The other method is to use intentionally doped samples where the QDs are initially filled with a random number of electrons from the background doping. ${ }^{6}$ The average number of excess electrons depends on the arbitrary number of dopants in the close

\footnotetext{
${ }^{a)}$ Electronic mail: freka@ifm.liu.se

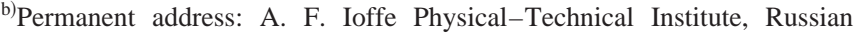
Academy of Sciences, 194021, Polytechnicheskaya 26, St. Petersburg, Russia.
}

vicinity of the investigated QD, but it can be reduced by means of photodepletion. The main drawback of this method is that the initial number of excess electrons is random and, hence, hardly controllable.

In the present letter, we report on the sensitivity of the capture rate of excess electrons into the SAQD on the effective diffusivity of photoexcited carriers in the barrier in lowdot-density samples. The effective diffusivity is not only dependent on the crystal temperature ${ }^{7}(T)$ but also on the excess energy of the carriers imposed at the excitation, ${ }^{8,9}$ defined as $\hbar \omega_{\text {photon }}-E_{g}^{\text {barrier }}$, where $\hbar \omega_{\text {photon }}$ is the excitation energy and $E_{g}^{\text {barrier }}$ is the QD barrier band-gap energy. We show that in the case of above-barrier band-gap excitation $\left(\hbar \omega_{\text {photon }}>E_{g}^{\text {barrier }}\right)$, the spectrum of the QDs is indeed very sensitive to the excitation energy and crystal temperature: Charged excitons are created just at certain excitation energies and temperatures. This can be used as an alternative tool, in addition to the two previously mentioned, to study the PL of charged excitons in QDs, whereby no special sample design is needed. The average number of excess electrons in the QD can be controlled by tuning $\hbar \omega_{\text {photon }}$ and/or $T$.

The samples studied were grown by molecular-beam epitaxy on semi-insulating GaAs(100) substrates. On top of a $100 \mathrm{~nm}$ GaAs layer, lens-shaped QDs are formed from about 1.7 InAs ML deposition. With a special growth technique to reduce the dot size ${ }^{2}$ and size distribution, the QDs were covered with $100 \mathrm{~nm} \mathrm{GaAs}$ for protection. A certain sample region of low-InAs deposition, and thereby a very low-dot density, ${ }^{1}$ was chosen for our study, where the interdot spacing was estimated to be about $10 \mu \mathrm{m}$, well exceeding the resolving power of the micro-PL setup used.

The QDs were studied by means of a conventional diffraction-limited micro-PL setup. To excite the QDs, a cw Ar laser pumped Ti-Sp laser tunable between 700 and 900 $\mathrm{nm}$ was used with an excitation power adjusted by the use of neutral filters. The beam of the Ti-Sp laser was focused on the sample surface by a microscope objective through a thin optical window of the continuous-flow cryostat. The tem- 


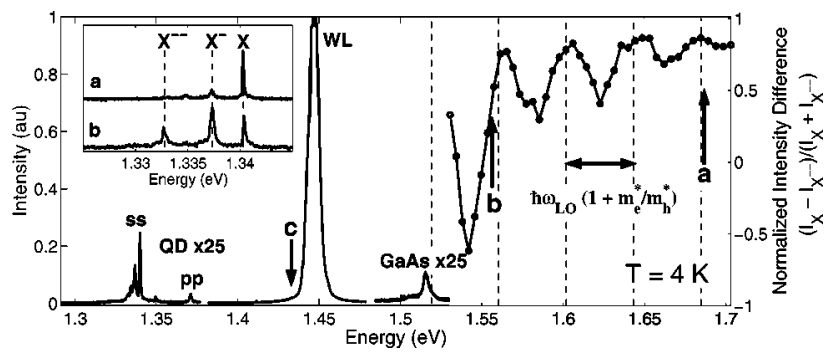

FIG. 1. Left-hand part shows PL spectra taken at $P_{\mathrm{ex}}=5 \mathrm{nW}, \hbar \omega_{\text {photon }}$ $=1.686 \mathrm{eV}$ and $T=4 \mathrm{~K}$ of the GaAs, the WL, and a single QD with the emissions originating from the ss ground state and the $p p$ excited state. The right-hand part shows the normalized intensity difference $\left(I_{X}-I_{X^{--}}\right) /\left(I_{X}\right.$ $+I_{X^{--}}$) between the two peaks denoted $X$ and $X^{--}$in the QD ground state vs the excitation energy $\hbar \omega_{\text {photon }}$, taken at $P_{\text {ex }}=10 \mathrm{nW}$. The three arrows marked correspond to different excitation energies (a) $\hbar \omega_{\text {photon }}=1.686 \mathrm{eV}$, (b) $\hbar \omega_{\text {photon }}=1.557 \mathrm{eV}$, and (c) $\hbar \omega_{\text {photon }}=1.433 \mathrm{eV}$. Five dashed lines in the right-hand part mark the excitation energies at which the electron's part of the excess energy is an integer times $\hbar \omega_{\mathrm{LO}}$, starting from the GaAs barrier band gap at $1.519 \mathrm{eV}$. A magnified region of the QD ground state is shown in the inset at two different excitation energies [(a) and (b)] above the GaAs band gap.

peratures used in the experiments ranged from 4 to $40 \mathrm{~K}$. The laser beam could be focused on the sample surface down to a spot size of $2 \mu \mathrm{m}$ in diameter. The luminescence signal was collected by the same objective and dispersed by a single-grating $0.45 \mathrm{~m}$ monochromator combined with a $\mathrm{LN}_{2}$-cooled Si charged-coupled-device camera. The spectral resolution achieved in the region of the studied PL was 0.15 $\mathrm{meV}$. Low-excitation power $P_{\mathrm{ex}} \leqslant 10 \mathrm{nW}$ was used, when exciting above the barrier band gap, to prevent local sample heating.

A magnified image of the sample surface was projected on a video camera, allowing precise control of the laser position and focusing. Since any change in the temperature causes a sample drift, special grids on the sample were used as a reference to locate the desired dot.

Several QDs have been studied. Most of them exhibit a similar behavior, as represented by the spectra of the QD shown in the present letter. The excitation energy dependence of the PL spectrum of a selected single QD at $T$ $=4 \mathrm{~K}$ is demonstrated in Fig. 1. The left part of Fig. 1 shows the PL emission from the GaAs barrier at $1.515 \mathrm{eV}$, the wetting layer (WL) at $1.447 \mathrm{eV}$, and the QD at $1.371 \mathrm{eV}(p p)$ and $\sim 1.340 \mathrm{eV}(s s)$. The ss emission corresponds to the QD ground-state recombination (both electrons and holes in the $s$ shell) and the $p p$ emission originates from the first-excitedstate recombination (both electrons and holes in the $p$ shell). The importance of diffusion of photogenerated carriers is readily seen in Fig. 1. Despite the fact that essentially all carriers are generated in the thick $(200 \mathrm{~nm}) \mathrm{GaAs}$ barrier, the emission from the thin $(<1 \mathrm{~nm}) \mathrm{WL}$ is two orders of magnitude stronger than the GaAs emission, indicating a strong diffusion from the GaAs layer to the WL. A magnified region of the ground-state emission is shown as an inset in Fig. 1 at an excitation energy of (a) $\hbar \omega_{\text {photon }}=1.686 \mathrm{eV}$ and (b) $\hbar \omega_{\text {photon }}=1.557 \mathrm{eV}$. Extra attention should be paid to the two peaks denoted $X$ and $X^{--}$since their intensities $\left(I_{X}\right.$ and $I_{X^{--}}$) exhibit a mutual competition, i.e., for a given excitation energy with a resulting maximum PL intensity of $I_{X}$ corresponds to a minimum of $I_{X^{--}}$, and vice versa. Accordingly, there is a clear oscillatory behavior of $I_{X}$ and $I_{X^{--}}$

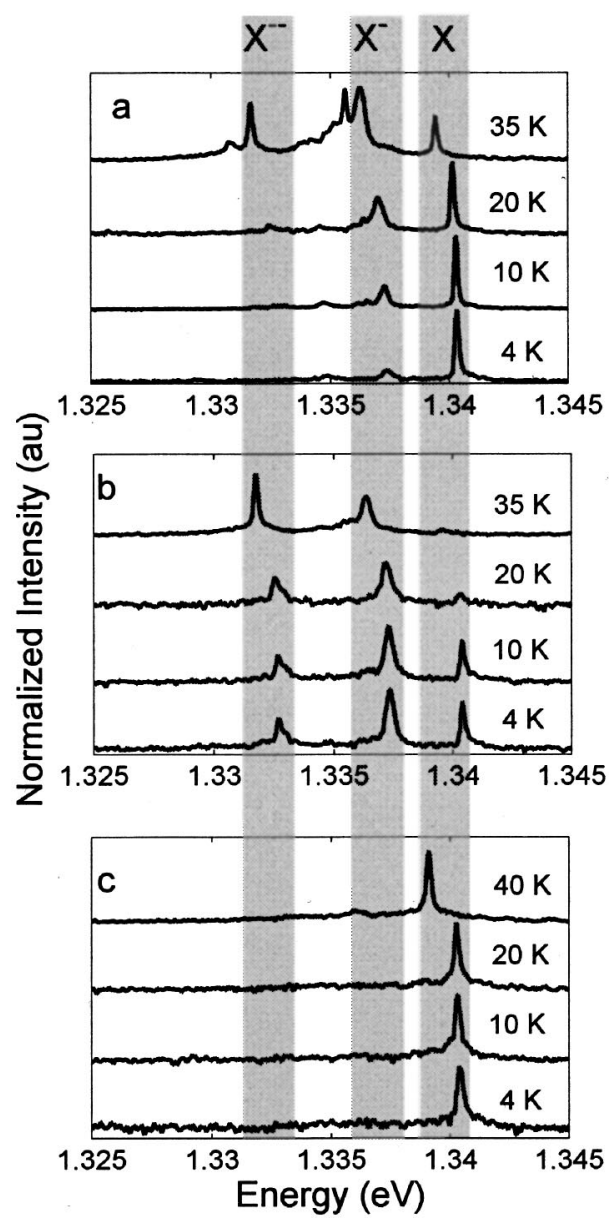

FIG. 2. Temperature dependence for a single QD PL spectrum measured with (a) $\hbar \omega_{\text {photon }}=1.686 \mathrm{eV}$ and $P_{\text {ex }}=5 \mathrm{nW}$, (b) $\hbar \omega_{\text {photon }}=1.557 \mathrm{eV}$ and $P_{\text {ex }}=5 \mathrm{nW}$, and (c) $\hbar \omega_{\text {photon }}=1.433 \mathrm{eV}$ and $P_{\mathrm{ex}}=30000 \mathrm{nW}$, respectively. All spectra are normalized to the maximum intensity and shifted vertically. The shaded areas mark the energy ranges for the three peaks in the relevant temperature interval used in this study $(4-40 \mathrm{~K})$.

with respect to the excitation energy $\hbar \omega_{\text {photon }}$, as shown in the right part of Fig. 1. Three different excitation energies [corresponding to the three arrows, (a), (b), and (c)] have been employed in the inset of Fig. 1 and in Fig. 2. The dashed vertical lines, starting at $E_{g}^{\mathrm{barrier}}=1.519 \mathrm{eV}$ for bulk GaAs, are separated by $41.4 \mathrm{meV}$, corresponding to $\hbar \omega_{\mathrm{LO}}\left(1+m_{e}^{*} / m_{h}^{*}\right)$, where $\hbar \omega_{\mathrm{LO}}=36 \mathrm{meV}$ is the GaAs longitudinal optical (LO) phonon energy and $m_{e}^{*}=0.067 m_{0}$ $\left(m_{h}^{*}=0.45 m_{0}\right)$ is the electron (heavy-hole) effective mass. ${ }^{8,9}$ Consequently, the observed periodicity of the intensities corresponds to the excitation energies $\hbar \omega_{\text {photon }}$ at which the electron's part of the excess energy in GaAs is exactly an integer times $\hbar \omega_{\mathrm{LO}}$, assuming parabolic band dispersion. ${ }^{10}$

The oscillating dependence of the QD exciton intensity on the excitation energy $\hbar \omega_{\text {photon }}$ can be explained in terms of the well-known phonon relaxation processes of carriers. ${ }^{10}$ The excess energy of the photogenerated carriers is essentially released either by a fast ${ }^{9}$ (few ps) emission of LO phonons or by a much slower emission of acoustic phonons. If the excess energy exactly fits with an integer number of $\hbar \omega_{\mathrm{LO}}$, the excess energy is very quickly released, whereby the carrier becomes motionless at the band edge shortly after generation. On the other hand, if the excess energy does not fit with an integer number $\hbar \omega_{\mathrm{LO}}$, it takes a much longer time 
to release the energy remaining after the LO-phonon emission. During this additional time, the carrier is able to move a longer distance in the crystal. In other words, excess energies that fit (do not fit) with $\hbar \omega_{\text {LO }}$ will result in a low (high) effective diffusivity. Carriers with a higher diffusivity will move in a spatially larger region and are thus more likely to pass in the near vicinity of a strong capturing center (a quantum well or a QD) and become trapped. Due to different masses, the excess energy is distributed in a nonequal way between the electrons and the heavy holes. Accordingly, during an initial state the capturing center will primarily trap the high-diffusivity carriers, but subsequently the attraction of low-diffusivity carriers of opposite charge will be increased due to the Coulomb interaction with the trapped highdiffusivity carriers. The steady-state conditions at continuous excitation require an equal capture probability of electrons and holes. This condition is thus precisely fulfilled when the capturing center accommodates a certain number of extra carriers of higher diffusivity.

The fact that the oscillations of $I_{X}$ and $I_{X^{--}}$perfectly correspond to the oscillation of the electron excess energy, ${ }^{10}$ combined with the fact that the theoretical models of lensshaped SAQDs predict only extra electrons to be bound to the excitons, ${ }^{11}$ implies that the peaks $X, X^{-}$, and $X^{--}$can be assigned as the pure ground-state exciton, the exciton plus one extra electron, and the exciton plus two extra electrons, respectively. A more-detailed theoretical treatment of the peak assignments is developed separately. ${ }^{12}$

The excess energy of the heavy holes will never reach $\hbar \omega_{\mathrm{LO}}$ in the excitation energy region under study, leading to a monotonous increase of the heavy-hole diffusivity. This could explain the decreasing amplitude of the intensity oscillations, to approach a predominant single $X$ line, upon increasing the excitation energy $\hbar \omega_{\text {photon }}$.

Another way to increase the effective diffusivity of the carriers is to increase the temperature $T{ }^{7}$ Since the electron mass is about seven times lighter than the heavy-hole mass in GaAs, the electrons gain a higher diffusivity than the heavy holes, when the temperature is increased. The PL spectra shown in Fig. 2 demonstrate the temperature dependence at three different excitation energies $\hbar \omega_{\text {photon }}$. Due to the small thermal shift of the GaAs band gap, the excitation energy $\hbar \omega_{\text {photon }}$ was shifted by the same amount in the cases when $\hbar \omega_{\text {photon }}>E_{g}^{\text {barrier }}$ to keep the excess energy imposed by the photoexcitation fixed. However, in the temperature region under study $(4-35 \mathrm{~K})$, this shift is very small $(<0.5$ $\mathrm{meV}$ ) and should not essentially affect the spectrum - even if not compensated. An analogous shift is also observable for the QD luminescence, as can be seen in Fig. 2. In Fig. 2(a), the excitation energy $\hbar \omega_{\text {photon }}$ is chosen [as marked by arrow (a) in Fig. 1] to give solely the pure exciton $X$ at $4 \mathrm{~K}$. It is clear that when the temperature is increased there is a successive transfer of the intensity from the $X$ line to the $X^{-}$and $X^{--}$lines at lower energy, indicating an increased probability that the QD becomes charged. At $35 \mathrm{~K}$ the three lines have comparable intensity. Further, in Fig. 2(b) the excitation energy $\hbar \omega_{\text {photon }}$ is chosen [as marked by arrow (b) in Fig. 1] to give comparable intensities for the three peaks at 4 $\mathrm{K}$. At $35 \mathrm{~K}$, the spectrum is dominated by $X^{--}$, while $X$ has vanished. The monotonous decrease of $X$ and the corre- sponding increase of $X^{--}$shows that an increased temperature effectively fills the QD with extra electrons.

To finally confirm that the temperature effect is due to an increased diffusivity and not due to an activation of localized electrons (for example, electrons localized at impurities caused by unintentional background doping), ${ }^{6}$ the sample was excited with an excitation energy $\hbar \omega_{\text {photon }}$ below the WL emission [corresponding to arrow (c) in Fig. 1], while the temperature was increased [Fig. 2(c)]. Since the carriers in this case are excited to localized states of the WL and, hence, are totally immobile, no diffusion takes place in either the GaAs or the WL, and accordingly no peak except the pure exciton $X$ is expected in the spectrum. If the extra electrons, on the other hand, are due to electron activation, the spectrum should qualitatively show the same temperature behavior as for excitation energies $\hbar \omega_{\text {photon }}>E_{g}^{\text {barrier }}$. Obviously, the spectra in Fig. 2(c) are temperature independent, except for a similar redshift as monitored in Figs. 2(a) and 2(b). This fact supports our explanation presented for the exciton line redistribution at elevated temperatures in terms of increased photogenerated carrier diffusivity.

In conclusion, we have demonstrated that a single QD photoluminescence spectrum is very sensitive to excitation energy and crystal temperature. A qualitative explanation is given in terms of the well-known phonon-relaxation processes that may cause different effective diffusivities of electrons and holes, resulting in QDs charged by one or more extra electrons. This important fact should always be taken into consideration when performing nonresonant excitation on low-dot-density SAQDs. Furthermore, this can be used as an effective tool to create charged excitons with precise control of the average number of excess electrons, requiring no special sample design or doping. The simplicity of charging the dot by tuning the excitation energy and/or temperature may simplify future studies of phenomena involving charged excitons in QDs.

One of the authors (E.S.M.) gratefully acknowledges the financial support of the Swedish Institute within the Visby Programme.

${ }^{1}$ D. Leonard, K. Pond, and P. M. Petroff, Phys. Rev. B 50, 11687 (1994). ${ }^{2}$ J. M. Garcia, T. Mankad, P. O. Holtz, P. J. Wellman, and P. M. Petroff, Appl. Phys. Lett. 72, 3172 (1998).

${ }^{3}$ A. Gustafsson, M. E. Pistol, L. Montelius, and L. Samuelsson, J. Appl. Phys. 84, 1715 (1998).

${ }^{4}$ P. Hawrylak, Phys. Rev. B 60, 5597 (1999); A. Wojs and P. Hawrylak, ibid. 55, 13066 (1997); R. J. Warburton, B. T. Miller, C. S. Durr, C. Bodefeld, K. Karrai, J. P. Kotthaus, G. Medeiros-Ribeiro, P. M. Petroff, and S. Huant, ibid. 58, 16221 (1998).

${ }^{5}$ M. Baier, F. Findeis, A. Zrenner, M. Bichler, and G. Abstreiter, Abstracts of the 25th International Conference on Physics of Semiconductor, Osaka, Japan (2000), pp. 207 (unpublished).

${ }^{6}$ A. Hartmann, Y. Ducommun, E. Kapon, U. Hohenester, and E. Molinari, Phys. Rev. Lett. 84, 5648 (2000); A. Hartmann, Y. Ducommun, M. Bachthold, and E. Kapon, Physica E (Amsterdam) 7, 461 (2000).

${ }^{7}$ H. W. Yoon, D. R. Wake, and J. P. Wolfe, Phys. Rev. B 46, 13461 (1992); H. Hillmer, A. Forchel, S. Hansmann, M. Morohashi, E. Lopez, H. P. Meier, and K. Ploog, ibid. 39, 10901 (1989).

${ }^{8}$ J. P. Wolfe, H. W. Yoon, D. R. Wake, and H. Morkoc, Semicond. Sci. Technol. 7, B240 (1992).

${ }^{9}$ H. W. Yoon, D. R. Wake, and J. P. Wolfe, Phys. Rev. B 54, 2763 (1996).

${ }^{10}$ R. W. Shaw, Phys. Rev. B 3, 3283 (1971); A. Nakamura and C. Weisbuch, Solid-State Electron. 21, 1331 (1978).

${ }^{11} \mathrm{Ph}$. Lelong and G. Bastard, Solid State Commun. 98, 819 (1996).

${ }^{12}$ E. S. Moskalenko, K. F. Karlsson, P. O. Holtz, B. Monemar, W. V. Shoenfeld, J. M. Garcia, and P. M. Petroff (unpublished). 